\title{
Iron at the Galactic Centre
}

SIR - The neighbourhood of the Galactic Centre is a region of considerable astronomical interest. An innermost core of diameter $\sim 300$ pc emits high fluxes of radiation over a wide range of wavelengths. Recent X-ray studies of the Galactic Centre using the Japanese Astronomical satellite Ginga lend strong support to the idea that $10^{3}-10^{4}$ supernovae have exploded in this region over the past 100,000 years ${ }^{1,2}$. Furthermore, the ejecta from such explosions may have led to a localized enhancement of X-ray line radiation at $6.7 \mathrm{keV}$, corresponding to an increased concentration of iron ${ }^{2}$. The relative abundance of iron in this region appears to be in excess of values in the Sun by a factor of about 2 .

This observed excess of iron, if it arose

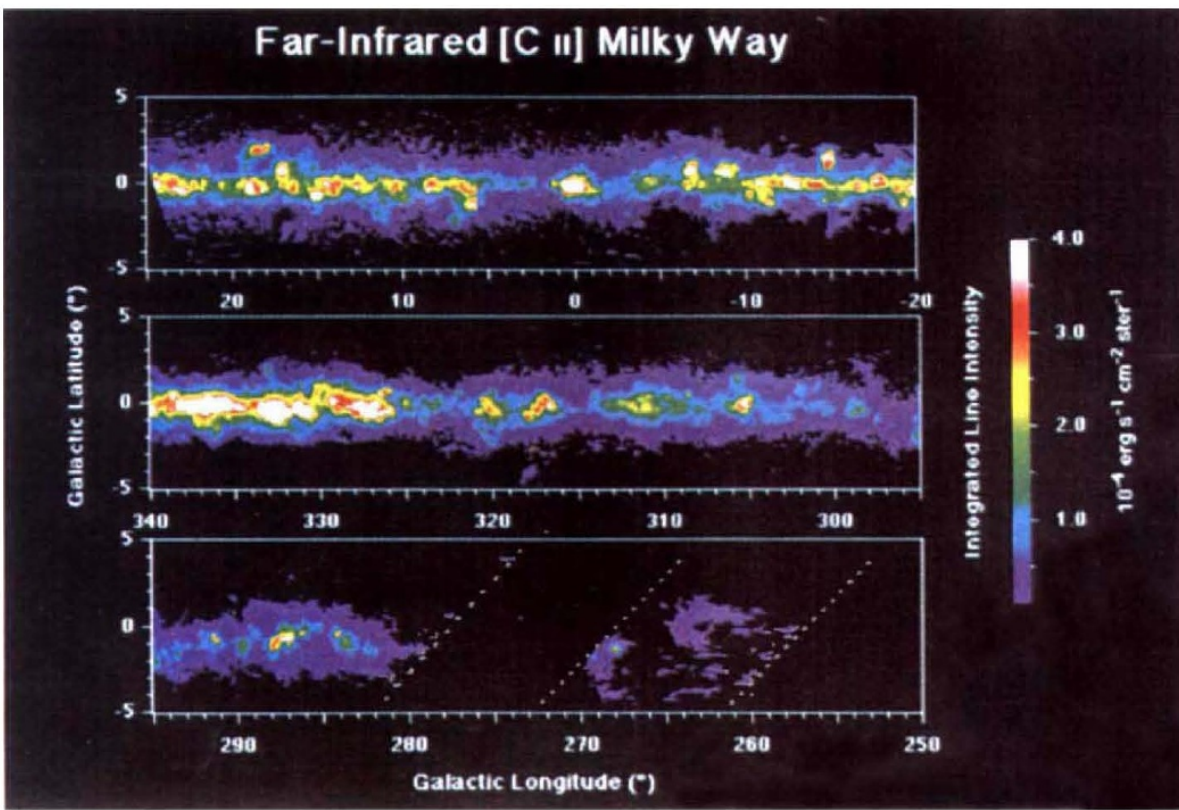

Map of the galactic plane in $158 \mu \mathrm{m} \mathrm{C}$ ॥ line radiation contours.

from supernovae, is likely to be in the form of solid particles, probably as iron 'whiskers' ${ }^{3,4}$. Such particles possess high values of the far infrared absorption coefficient that could lead to important observable effects ${ }^{5}$. The calculated mass absorption coefficient for cryogenic iron whiskers of radius $0.01 \mu \mathrm{m}$ and of various lengths peaks at wavelengths in the range $100-300 \mu \mathrm{m}$, reaching maximum values of a few times $10^{6} \mathrm{~cm}^{2} \mathrm{~g}^{-1}$ (see ref. 5 for details of the calculations).

For such values of the absorption coefficient, and with reasonable assumptions about the energy density of the radiation field in the Galactic Centre region, the average time for supernova grains to be expelled due to radiation pressure from an inner core region of diameter $300 \mathrm{pc}$ is about $10^{7} \mathrm{yr}$. This is well in excess of the $10^{5}$-yr timescale during which about 3,000 supernovae seem to have exploded, according to the recent X-ray data, which is also the dynamical timescale for the Galactic Centre plasma ${ }^{2}$. Because an average type II supernova produces about 0.1 solar masses of iron, the mass of iron contained within, say, a sphere of radius $R$ $\approx 150 \mathrm{pc}$ around the centre, is about 300 solar masses. The average column density of iron through this region is therefore $\sim 6 \times 10^{35}(3 R / 2)\left(4 \pi R^{3} / 3\right) \simeq 10^{-6} \mathrm{~g} \mathrm{~cm}^{-2}$.

These considerations can be seen in the light of the important implications of recent far-infrared observations made using balloons and satellites. A surprising discovery has been the depressed intensities found for both the C II $(158 \mu \mathrm{m})$ and the N II $(205 \mu \mathrm{m})$ lines at the precise location of the Galactic Centre, compared with the much stronger peaks of emission

that occur at galactic longitude $=330-$ $340^{\circ}$ (refs 6,7 ). This situation also contrasts markedly with the data for the $\mathrm{CO}$ line at $2.6 \mathrm{~mm}$, which shows its strongest intensity at the Galactic Centre itself. The figure shows this effect quite strikingly in the case of the $\mathrm{C}$ II emission contours from the galactic plane ${ }^{7}$. Iron particles of the kind thought to form in supernova explosions would clearly depress the strengths of both the $\mathrm{C}$ II and the $\mathrm{N}$ II lines to the

1. Koyama, K. etal. Nature 339, 603-605(1989)

2. Koyama, K. \& Yamauchi, S. AlP Conf. Proc. 278: Back to the Galaxy 107-114 (American Institute of Physics, New York, 1993).

3. Hoyle, F. \&Wickramasinghe, N. C. Astrophys. Space Sci. 147, 245-256 (1988)

4. Wickramasinghe, N. C., Wickramasinghe, A. N. \& Hoyle, F. Astrophys. Space Sci. 193, 141-144 (1992).

5. Wickramasinghe, N. C. \& Hoyle, F. Astrophys. Space Sci. (in the press)

6. Bennett, C. L. \& Hinshaw, G. AlP Conf. Proc. 278: Back to the Galaxy 257-266 (American Institute of Physics, New York, 1993).

7. Nakagawa, T. et al. AlP Conf. Proc. 278: Back to the Galaxy 303 (American Institute of Physics, New York, 1993) desired extent, but would leave the $\mathrm{CO}$ line at $2.6 \mathrm{~mm}$ essentially untouched. Thus, the new far infrared data could be interpreted as further evidence for supernova-produced iron whiskers near the Galactic Centre.

N. C. Wickramasinghe

School of Mathematics,

University of Wales College of Cardiff,

Cardiff CF2 4AG, UK

H. Okuda

Laboratory for Infrared Astronomy,

Institute of Space and Aeronautical

Studies, Sagamihara, Kanagawa, Japan

\section{Gelatinase A and APP}

SIR - We previously reported ${ }^{1}$ that APP, a precursor of the $\beta / \mathrm{A} 4$ amyloid peptide, has a proteinase inhibitor domain for gelatinase $\mathrm{A}$, a member of the matrix metalloproteinase (MMP) family, and that the enzyme has $\alpha$-secretase-like, $\beta$ AP-hydrolytic activity. These facts suggest the possibility that gelatinase A may function in the metabolism of APP as $\alpha$-secretase or $\beta \mathrm{AP}$-degrading enzyme. Walsh et al. in Scientific Correspondence ${ }^{2}$ reported that the rat adrenal pheochromocytoma cell line PC-12 does not secrete gelatinase $\mathrm{A}$, and that addition of the metalloproteinase inhibitor TIMP-1, a glycoprotein of $M_{\mathrm{r}} 28,000$, into the culture of PC-12 cells does not affect the secretion of APP. They therefore concluded that gelatinase $\mathrm{A}$ is unlikely to be $\alpha$-secretase in these cells.

We have now carried out similar experiments using the same cell line but have obtained different results. Analysis of serum-free conditioned medium of PC-12 cells by substrate gel SDS-PAGE (zymography) shows that $\mathrm{PC}-12$ cells secrete the latent proforms of gelatinases $A$ and $B$ (see figure). When the cells are induced by nerve growth factor to differentiate into neuronal cells, the secretion of both enzymes, especially gelatinase A, is markedly stimulated. This treatment stimulates not only the secretion of the proenzymes but also their conversion into active forms. The discrepancy between our results and those of Walsh et al. ${ }^{2}$ may result from differences in experimental methods. Using northern blotting, we find that messenger RNAs encoding gelatinase $A$ and its natural inhibitors, TIMP-1 and TIMP-2, are constitutively expressed in human brain tissue (data not shown), suggesting that gelatinase A may play a fundamental role in the central nervous system.

In agreement with Walsh et al. ${ }^{2}$, we observed no significant effect of TIMP-1 on the APP secretion when it was added into the culture of PC-12 cells. But in $\mathrm{PC}-12$ cells $^{3}$ and human neuroglioma (H4) 